\title{
Correction to: Next-generation sequencing analysis of receptor-type tyrosine kinase genes in surgically resected colon cancer: identification of gain-of-function mutations in the RET proto-oncogene
}

Duarte Mendes Oliveira ${ }^{1}$, Katia Grillone', Chiara Mignogna², Valentina De Falco ${ }^{4}$, Carmelo Laudanna', Flavia Biamonte ${ }^{1}$, Rosa Locane ${ }^{3}$, Francesco Corcione ${ }^{5}$, Massimiliano Fabozzi ${ }^{5}$, Rosario Sacco ${ }^{3}$, Giuseppe Viglietto ${ }^{1 *}$, ${\text { Donatella Malanga }{ }^{1 *} \text { and Antonia Rizzuto }}^{3}$

\section{Correction}

In the publication of this article [1], there is an error in Fig. 7. The minus and plus signals are inverted which impairs understanding of the results described.

Figure 7 should instead be read as indicated in this correction.

This has now been included in this erratum.

\begin{abstract}
Author details
${ }^{1}$ Department of Experimental and Clinical Medicine, University Magna

Graecia of Catanzaro, Campus Salvatore Venuta -Viale Europa, 88100

Catanzaro, Italy. ${ }^{2}$ Department of Health Sciences, University Magna Graecia of

Catanzaro, Campus Salvatore Venuta - Viale Europa, 88100 Catanzaro, Italy. ${ }^{3}$ Department of Medical and Surgical Sciences, University Magna Graecia of Catanzaro, Campus Salvatore Venuta - Viale Europa, 88100 Catanzaro, Italy. ${ }^{4}$ Department of Molecular Medicine and Medical Biotechnologies, University Federico II, Naples, Italy. ${ }^{5}$ OC Chirurgia Generale, Azienda Ospedaliera dei Colli, Naples, Italy.
\end{abstract}

Received: 18 April 2018 Accepted: 18 April 2018

Published online: 01 June 2018

\section{Reference}

1. Mendes Oliveira D, Grillone K, Mignogna C, et al. Next-generation sequencing analysis of receptor-type tyrosine kinase genes in surgically resected colon cancer: identification of gain-of-function mutations in the RET proto-oncogene. J Exp Clin Cancer Res. 2018;37:84. https://doi.org/10. 1186/s13046-018-0746-y

\footnotetext{
*Correspondence: viglietto@unicz.it; malanga@unicz.it

${ }^{1}$ Department of Experimental and Clinical Medicine, University Magna Graecia of Catanzaro, Campus Salvatore Venuta -Viale Europa, 88100 Catanzaro, Italy
} 


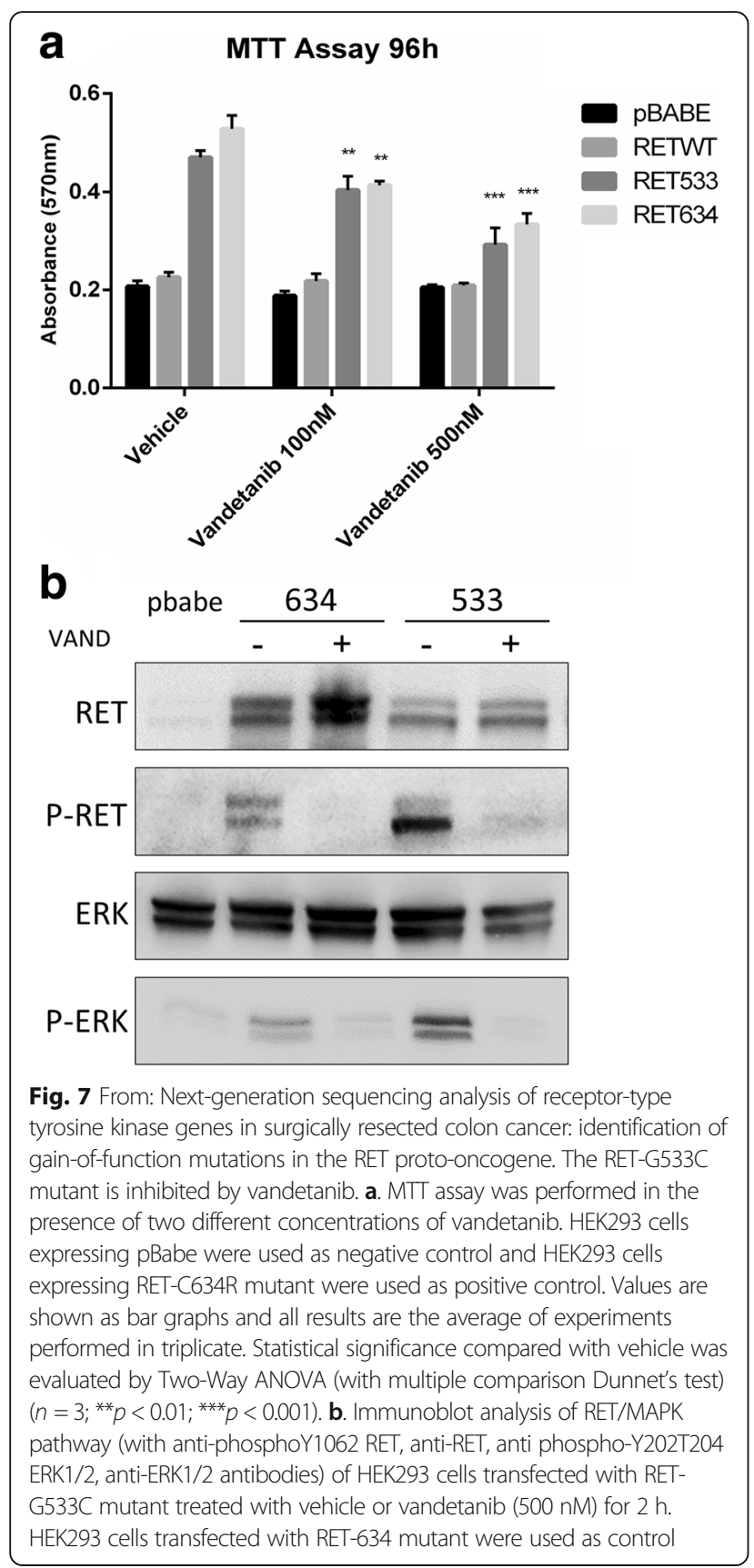

